\title{
The Effects of a Primary Nutritional Deficiency (Vitamin B Study)
}

\author{
Hugh D. Riordan, Nina Mikirova, Paul R. Taylor, Cindy A. Feldkamp, Joseph J. Casciari
}

The Riordan Clinic Research Institute, Riordan Clinic, Wichita, USA.

Email: nmikirova@riordanclinic.org

Received July $11^{\text {th }}, 2012$; revised August $10^{\text {th }}, 2012$; accepted August $17^{\text {th }}, 2012$

\begin{abstract}
It has long been understood there is a link between a nutrient deficiency and visually measurable health. In separate studies, Sprague Dawley or Fischer 344 rats were fed diets lacking 100\% of one B vitamin until a single mortality occurred. Vitamins studied were thiamin, riboflavin, niacin, pyridoxine, pantothenic acid, cobalamin and folate. Body mass, food consumption and images were recorded at weekly intervals. Rats were euthanized and liver, kidney, heart and brain samples for histological analysis were prepared. Liver and brain tissues were chosen as markers because of the high need of that organ for B vitamins and the likelihood of secondary lesion development. Correlation of deficiency symptoms with one missing nutrient was explored.
\end{abstract}

Keywords: Essential Nutrients; B Vitamins; Deficiency Symptoms; Animal Study

\section{Introduction}

B vitamins are essential nutrients that support carbohydrate metabolism, enhance immune system function, and promote cell growth. For example, riboflavin (vitamin B2), niacin (vitamin B3), and pantothenic acid (vitamin B5) share a role with thiamine (vitamin B1) as essential coenzymes for energy metabolism.

Phosphorylated forms of thiamine (vitamin B1) catalyze the formation of acetyl-coenzyme A, succinyl-coenzyme $\mathrm{A}$, and amino acid derivatives that play critical roles in cellular energy production [1,2] and the pentose phosphate pathway. It is also required for the synthesis of the nucleic acids, DNA and RNA, and the niacin-containing coenzyme NADPH, which is essential for a number of biosynthetic reactions [3-5]. Thiamin deficiency affects the cardiovascular, nervous, muscular, and gastrointestinal systems. In mammals, deficiency results in Korsakoff's syndrome, optic neuropathy, and a disease called beriberi that affects the peripheral nervous (polyneuritis) and/or cardiovascular system. Thiamine deficiency has a potentially fatal outcome if it remains untreated.

Niacin (B3) occur in biological systems as free acids (as nicotinamide) and in the form of coenzymes, nicotinamide adenine dinucleotide (NAD) and nicotinamide adenine dinucleotide phosphate (NADP). As many as 200 enzymes require the niacin coenzymes, NAD and NADP, mainly to accept or donate electrons for redox reactions. NAD functions most often in energy producing reactions involving the degradation (catabolism) of carbohydrates, fats, proteins, and alcohol $[5,6]$.

Pantothenic acid (vitamin B5) is a component of coenzyme A (CoA), an essential coenzyme in a variety of reactions that sustain life. CoA is required for chemical reactions that generate energy from food (fat, carbohydrates, and proteins). The synthesis of essential fats, cholesterol, and steroid hormones requires CoA, as does the synthesis of the neurotransmitter, acetylcholine, and the hormone, melatonin. Symptoms of pantothenic acid deficiency include impaired energy production, irritability, fatigue, and apathy [7].

Pyridoxine (vitamin B6) is a coenzyme in over onehundred essential metabolic reactions, particularly those associated with metabolism of amino acids [8-12]. It is essential for normal brain development and function, as it aids in the production of neurotransmitters such as serotonin, dopamine, norepinephrine and gamma-aminobutyric acid [9]. Vitamin B6 deficiency is associated with growth depression, mild microcytic hypochromic anemia, convulsive seizures and calcium oxalate nephrosis [11].

Folic acid (vitamin B9) coenzymes appear to mediate the transfer of one-carbon units in reactions critical to the metabolism of nucleic acids and amino acids [13]. The synthesis of DNA from its precursors (thymidine and purines) is dependent on folate coenzymes, which are also necessary for the metabolism of methionine. Rapidly dividing cells, such as those in the bone marrow, are most vulnerable to the effects of folic acid deficiency. Common 
symptoms of folate deficiency include diarrhea, macrocytic anemia with weakness or shortness of breath, nerve damage and limb numbness (peripheral neuropathy, pregnancy complications, mental confusion, forgetfulness or other cognitive declines, mental depression, headaches and heart palpitations $[14,15]$. The effects of folic acid deficiency on DNA synthesis and repair may also lead to cancer development [16].

Cobalamin (vitamin B12) has the largest and most complex chemical structure of all the vitamins. It is unique among vitamins in that it contains a metal ion, cobalt. In mammals, cobalamin is a cofactor for two enzymes: one required for methionine synthesis and one for thymadylate synthesis. Both of these, in turn, are critical in DNA and RNA synthesis [17]. Vitamin B12 deficiency can potentially cause severe and irreversible damage to the brain and nervous system [18].

Vitamin B2 is required for a wide variety of cellular processes. Riboflavin is the central component of the cofactors flavin adenine dinucleotide (FAD) and flavin mononucleotide (FMN), and is therefore required by all flavoproteins. Flavocoenzymes participate in redox reactions in numerous metabolic pathways and are critical for the metabolism of carbohydrates, fats, and proteins. FAD is part of the electron transport (respiratory) chain, which is central to energy production [19].

As the several components of the diet are important for the supply and turnover of single carbon compounds in the body, such as methionine, choline, vitamin B12 and folic acid, we concluded that the specific requirement for one of these nutrients cannot be established unless the levels of other nutrients are taken into consideration.

The metabolic pathways of choline, methionine, methyl folate and vitamin B12 are interdependent. The existing data suggest a strong interaction between vitamin B12, choline deficiencies and folate status. Choline is utilized as a source of methyl groups in the absence of folate. Vitamin B12 is also important for folic acid dependent reactions of intermediary metabolism. The transfer of methyl groups from folic acid metabolite to homocysteine in the single carbon metabolic pathway, forming methionine is catalyzed by methionine synthase, a vitamin B12 dependent enzyme. Most species normally do not require dietary folic acid because of their ability to utilize intestinal microbial metabolites. The folic acid requirement increases if there is deficiency of choline and vitamin B12 in the diet.

In an effort to document the importance of B-vitamins, we studied rats given vitamin deficient diets. Our results, documented in the present manuscript, show how rat growth, appetite, and organ development are affected by depletion of any one B vitamin, and also show the ability of re-supplementation to reverse these effects.

\section{Methods}

Our work was broken down into three studies. In Study 1, we analyzed the effects of thiamin, pantothenic acid, pyridoxine or folate deficiency on male weanling Sprague Dawley rats (Charles River Laboratories). Fifteen rats, averaging 72.9 grams, were randomly separated into caging groups of three and acclimated for 24 hours. Pellets were fed bi-weekly, ad libitum. The feeding groups were AIN-93G control diet [20], and feeds deficient in one of the $\mathrm{B}$ vitamins listed above. Animals were handled daily for seven days to reduce stress of later blood sampling and were identified with an India ink tattoos applied to the tail. Reverse osmosis rural well water was provided free choice in sipper bottles. The 24 ” $\times 14$ ” $\times 16$ ” galvanized enhanced cages with ladder and resting ledge had a wire bottom to prevent ingestion of feces and litter [21]. A pelleted pine cat litter in the plastic tray under the cages controlled odor; while absorbing liquid waste. Flourescent lighting provided an 8 hours light/16 hours dark cycle. The feed, purchased from Dyets, Inc., Bethlehem, PA, was stored at $-4^{\circ} \mathrm{C}$ and offered in large crock bowls. A separate bowl was available for group snuggling. At study end, each rat was humanely euthanized in a $\mathrm{CO}_{2}$ chamber, liver and brain tissues were examined.

In Study 2 the effects of folate, choline, and vitamin B12 deficiencies on liver and brain development in young (five week old) male CFD rats were analyzed. In order to completely shut down the homocysteine metabolic pathway and fully visualize true folate deficiency, deficiencies of folate, choline, B12, and methionine are necessary. The addition of succinyl sulfathiazole disables the microflora production of B-vitamins in the gut. Diet \#517853 was designed for our purposes, combining the advantages of DyetDiet \#518753, the choline deficient L-amino acid diet and DyetDiet \#517777, the folate deficient L-amino acid diet [22]. The $1.7 \mathrm{mg} / \mathrm{Kg}$ methionine prolonged survival and the resultant pre-cancerous liver lesions [23-25]. Twelve rates averaging 141.7 grams mass, from Charles River Laboratories, were placed in three enhanced cage groups and fed a modified choline deficient L-Amino acid defined diet, Diet \#517853, with 3.7 mg/Kg methionine. The pellets and powder purchased from Dyets, Inc., were fed ad libitum until the first death. There were 2 cage groups with 4 each, as the controls. They were fed AIN-93G powder mixed on site until 8 weeks age, when after which AIN-93M was used. All study parameters were the same as previous, except alfalfa pellets were used as a litter [26,27] and the lighting was a 12 hours light/12 hours dark cycle. Blood samples for analysis were obtained from the thorax, after the portal vein was severed.

The intention of Study 3 was to repeat Study 2, as before, for half the animals, then reverse deficiency symptoms in the remaining half. Rats were gently euthanized 
with isoflourane.

The protocol of study was approved by Institutional Review Board. All animal protocols and experiments were approved by an Institutional Animal Care and Use Committee before the start of the study. Animals care and handling was in accordance with Office of Laboratory Animal Welfare (OLAW) and Animal Welfare Act (AWA) guidelines in effect at the time the research was conducted and was under the supervision of a veterinarian.

\section{Results}

\subsection{Study 1: Single Vitamin B Deficiency}

Figure 1 shows how deficiencies in each of four B vitamins affected rat growth.

Control rats ate roughly $21.3 \pm 2.0$ (SD) grams/day of food and grew at a rate of $7.1 \pm 0.4$ (SD) grams per day. They had a large amount of abdominal fat, pink adrenals and a large thymus. Livers excised from the control rats averaged 11.3 grams. One was of light color and fatty texture; the other two were a normal brownish-purple. Weight and food consumption data were normal. Figure 1 and Table 1 show how rat growth rates and appetites (food consumption rates) in B vitamin deficient rats compared to controls.

Thiamine deficiency led to significantly reduced growth and appetite. The thiamin deficient rats reached a weight plateau after two weeks and food consumption fell at or below beginning levels after a peak at 2 weeks. Hair loss, weakness, anorexia and depression followed. At study end, day 24, all study groups were physically active, except the thiamin deficient groups. These showed an ambling shuffle and intense weakness. Several thiamin deprived rats exhibited seizures prior to necropsy. Fur became coarse and brittle with some loosing up to $50 \%$ of

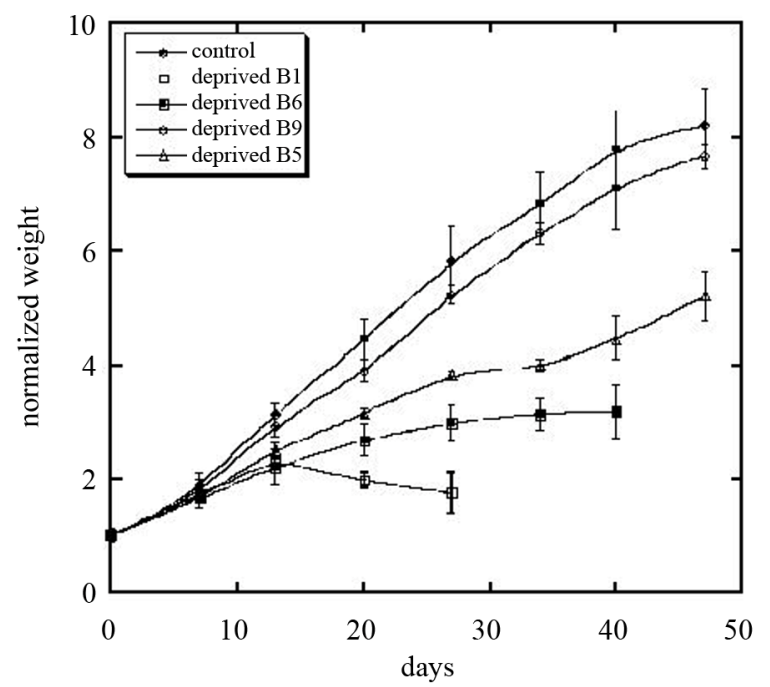

Figure 1. Growth rates of rats fed normal diet or diets deficient in B vitamins. hair. One liver was gray at necropsy; all others were normal color and texture, and average liver weight was 5.66 grams.

After 4 weeks, thiamin deficient rats were returned to normal diet, which resulted in improvement of appetite, food consumption and increasing in growth rate. Figure 2 shows the weight gain for control group of rats and for rats deprived vitamin B1 and returned to normal chow.

Pyroxidine deficient rats grew at roughly half the rate of controls, and had noticeably reduced appetites. These rats had painful inflammation of noses, ears, feet and tails. They carried their tails in a stiff, curled position and exhibited anorexia, weakness and depression. Alopecia developed after one week on deficient diet, possibly from

Table 1. Growth rates and food consumption rates of rats fed normal diets or diets deficient in $B$ vitamins. The ratio is the rate for deficient rats divided by that for controls. All errors are given as standard deviations.

\begin{tabular}{ccc}
\hline B1 & Growth & Appetite \\
\hline Control & $7.1 \pm 0.4 \mathrm{~g} /$ day & $21.3 \pm 2.8 \mathrm{~g} /$ day \\
Deprived & $1.1 \pm 1.0 \mathrm{~g} /$ day & $6.7 \pm 3.0 \mathrm{~g} / \mathrm{day}$ \\
Ratio & $0.16 \pm 0.15$ & $0.31 \pm 0.15$ \\
B5 & & \\
Control & $8.1 \pm 0.3 \mathrm{~g} /$ day & $25.6 \pm 3.6 /$ day \\
Deprived & $4.3 \pm 0.3 \mathrm{~g} /$ day & $15.4 \pm 3.1 /$ day \\
Ratio & $0.53 \pm 0.04$ & $0.60 \pm 0.15$ \\
B6 & & \\
Control & $7.1 \pm 0.4 \mathrm{~g} /$ day & $21.3 \pm 2.8 \mathrm{~g} /$ day \\
Deprived & $3.4 \pm 0.4 \mathrm{~g} /$ day & $14.0 \pm 5.9 \mathrm{~g} /$ day \\
Ratio & $0.49 \pm 0.06$ & $0.66 \pm 0.29$ \\
B9 & & \\
Control & $7.1 \pm 0.4 \mathrm{~g} /$ day & $21.3 \pm 2.8 \mathrm{~g} /$ day \\
Deprived & $6.6 \pm 0.3$ g/day & $20.8 \pm 4.9 \mathrm{~g} /$ day \\
Ratio & $0.93 \pm 0.06$ & $0.98 \pm 0.27$ \\
\hline
\end{tabular}

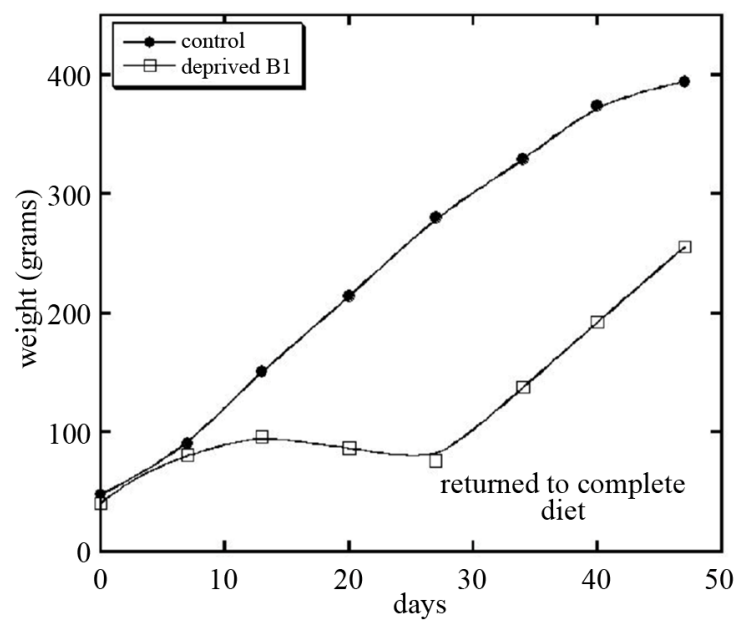

Figure 2. Growth rate of rats fed normal diet or diet deficient in vitamin $\mathbf{B} 1$. 
the rats ingesting hair from each other in an effort to obtain pyridoxine. Frequent grooming of the nose and tail were observed, as well as a distressed facial expression. At necropsy, one liver had an orange tint. The remaining samples were within normal limits for color and texture, and the average weight was 7.55 grams.

No illness, nervousness or deaths were found in any pantothenic deficient rats. However, they did have reduced growth and appetite, as shown in Figure 1 and Table 1. Typical, severe graying was observed in these rats. All had an ill and unthrifty look. One had inflammation of the prepuce from stress-induced over grooming. Much pathology was noted at the end of the study. Half of the livers were dark, mottled or nearly black. Average weight was 9.0 grams. Two rats had enlarged hearts. The thymus ranged from very small in 2 rats to excessively large in one individual. Only one had measurable abdominal fat. Adrenals ranged from normal pink to slightly dark to very dark red, and one rat was missing the left adrenal entirely.

The simple folate (B9) deprived rat diet included succinyl sulfathiazole to inhibit B vitamin synthesis in the gut. This was not adequate for any symptoms of deficiency to be seen. The growth and feed consumption curves were normal shape and only slightly depressed from that of the controls.

Rats deficient in niacin changed noticeably in terms of growth rates, and showed some unusual symptoms (data are not shown). The niacin deficient rats suffered symptoms such as dermatitis, yellow tinted hair, and bloody discharge, while the riboflavin deficient rats had minor alopecia, much finer hair coat than controls, and a temporary episode of bloody discharge from the mucous membranes.

\subsection{Study 2: Folate/Choline/B12 Deficiency}

Generally, the health of the deficient animals deteriorated over the eleven week course of the study. Initial symptoms were reduced weight gain. After two weeks, bleeding from the mucosa of the nose began to appear, and all deficient rats were affected by study's end. The deficient group also exhibited a 50\% incidence of hyperplastic lesions in the liver as a result of deficiency. This was an expected result in this model and could reach $100 \%$ with longer periods of deficiency using higher methionine levels. Others have noted the progression to tumors with longer deficiency periods.

At four weeks, deficient rats were not interested in the soft pellets, so the feeding protocol was modified. The best results were obtained by first offering animals frozen dry powder, which they would eat vigorously for two to five minutes, and then providing cold pellets. Little ingestion of food occurred during dark periods when rats tend to have greatest activity. Starting at seven weeks, with food intake critical, food was offered twice a day.
This increased consumption and temporary improvement in condition resulted. Observations at this time: petechial hemorrhages of the eyes, nostrils, toes and ears; eye color fading from deep red to a very pale pink; large amount of shed hair in control litter pan but very little in deficient litter pans; pale pink ears. At nine weeks, the study revealed extremely pale eyes and ears on weak, emaciated rats that weighed close to their original weights. Excessive face washing was the only activity besides the twice daily ventures to the food bowl. Deficient rats were wobbly in gait and tended to sit and stare when not otherwise active. Food consumption declined steadily, in spite of a rally of enthusiasm for eating.

The controls, at necropsy, were normal with healthy amounts of abdominal fat and typical size of organs. The comparative organ sizes for deficient rats are shown in Figure 3. Reduced sizes, relative to controls, of the liver $(0.55 \pm 0.2)$ and heart $(0.84 \pm 0.17)$ were statistically significant, while those in the brain $(0.96 \pm 0.15)$ were not. None of the rats in the deficient group had a measureable thymus. In some cases, abnormalities such as enlarged heart, plaque on the myocardium, liver lesions, and a smooth, avascular, mushy brain were observed. A smear was made of the exudate on the food bowls. Microscopic examination of smears from the exudate on food bowls revealed that these were primarily red blood cells. Necropsy of the nasal and oral cavities showed bleeding to originate in the nasal turbinate membranes, not the oral cavity.

\subsection{Study 3: Folate/Choline/B12 Deficiency with Re-Supplementation}

Choline/folate/chobalamin deficiency reduced rat growth rates, but this effect could be reversed by restoring the nutrient. This is shown in Figure 4, where the growth of

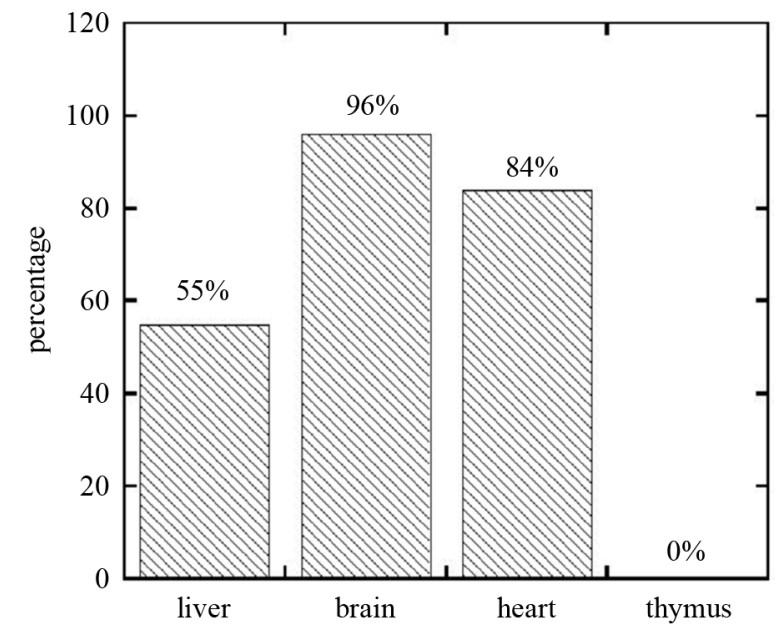

Figure 3. Effect of folate, choline and cobalamin depletion on organ development (percent mass of organ compared to control ). 
control and deficient rats are compared in two experiments, one where nutrient depletion was maintained and one where deficient rats were "re-supplemented" by providing normal chow after six weeks. In the re-supplementation experiment (Figure 4(b), Study 3), symptoms observed in the earlier study (Figure 4(a), Study 2) were exacerbated early; then paralleled the previous experiment. Symptoms in this repeat study were exacerbated early; then paralleled the previous study. Food consumption data was also parallel. Starting on day four, deficient rats showed soft, nearly black feces and evidence of diuresis. In some cases, rats had hemorrhage on front paws and appeared ill. During the depletion phase, reduced play, squabbling, loss of appetite, teeth grinding, over-grooming, depression, weakness, dehydration, hair loss, and nasal discharge were observed in some rats. Starting at week six, vitamin deficient rats were losing weight.

At week seven, some rats were euthanized so that tissue samples could be obtained and the rest were returned to complete rations. With the strong smell of folic acid present, they ate immediately. Squabbling among these weak, emaciated rats was observed. Small amounts were provided in three feedings per day for the first three days to prevent overindulgence and fatalities. Within five days after resupplementation, nasal discharge was less prevalent and all rats were looking healthier. Activity, eye color and hair coat all improved, as did appetite. Rat growth accelerated as the once deficient rats tended to make up ground to reach sizes closer to those of the control rats.

The comparative organ sizes for deficient rats at week seven (prior to re-supplementation) were reduced relative to controls. Reductions in size of the liver $(0.57 \pm 0.07)$

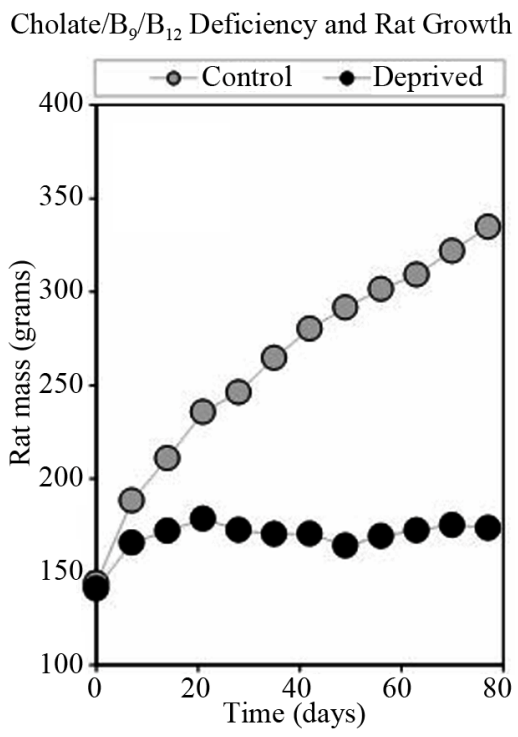

(a) and heart $(0.51 \pm 0.09)$ were statistically significant, and none of the rats in the deficient group had a measureable thymus. However, re-supplementation restored organ growth. Comparative organ sizes for re-supplemented rats increased in liver $(0.79 \pm 11)$, heart $(101 \pm 9)$ and thymus $(0.73 \pm 0.25)$ to values near unity.

\section{Discussion}

There are approximately 40 known nutrients essential for life. The body compensates for an imbalance, but health and well-being is severely compromised by a major or complete deficiency of any given nutrient. Our research clearly proves that a deficiency in a single B vitamin can stunt growth and produce significant adverse effects. Depression exhibited in thiamine or pyridoxine depleted rats are consistent with the roles these vitamin play in amino acid metabolism and the formation of key neurotransmi 他 - ters such as serotonin, melatonin, and dopamine $[28,29]$. Reductions in rat growth rates accompanying $\mathrm{B}$ vitamin deficiencies are consistent with the importance of B vitamins in cellular energy metabolism. Other adverse events observed in our study include hair loss, weakness, loss of appetite, inflammation, dermatitis, and bloody discharge. Necropsy showed reductions in liver and heart development, and absence of the thymus.

Many of the other nutrients in our studies resulted in one or more blocked or impaired pathways individually. Since methylation is a key component to gene expression, DNA synthesis, and non-essential amino acid synthesis it is a highly conserved and multifaceted pathway to prevent total blockage by a single nutrient deprivation.

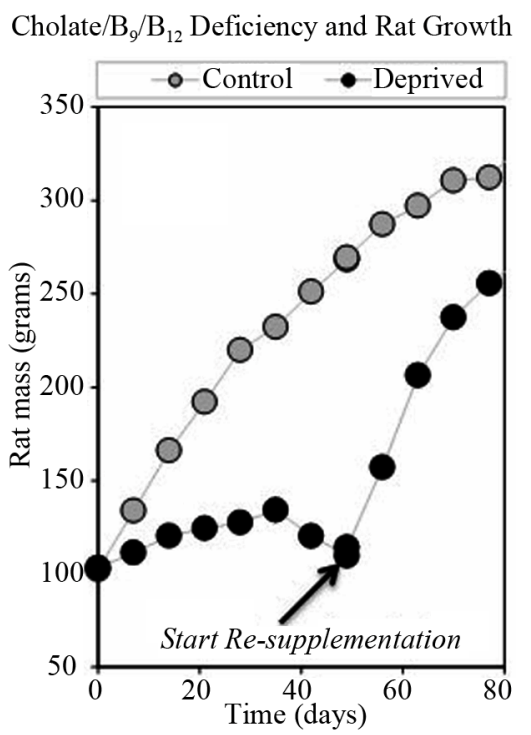

(b)

Figure 4. Growth rates of rats fed normal diets or diets deficient in folate, choline, and cobalamin. Graph (a) shows an experiment where deficiency was maintained; while graph (b) shows data when deprived rats were given normal chow starting after six weeks. 
A true state of deficiency arises when methionine, folate, choline and B12 are kept at zero; however, survival is only 4 weeks with inadequate time for development of liver lesions. When a minimal amount of methionine is added, survival is extended and pre-cancerous lesions can occur. Deficient Animals had 50\% incidence of precancerous lesions and no detectable adrenal glands.

Deficiencies in folic acid, choline and B12 can impair liver and pancreatic function and damage the intestinal tract. Other studies have shown that folic acid deficiency in rats causes the intestinal wall to become thin and lose the villi due to impaired DNA synthesis and reduced cell replication [30-32]. Loss of villi leads to poor absorption of the nutrients from food. The prolonged hemorrhage, reduced caloric intake, and inadequate absorption of all nutrients add up to cachexia, starvation and death. When complete diet is re-introduced to survivors, the cascade of deterioration very rapidly reverses as systems regenerate, although existing liver pathology may not be reversed. Behavioral studies to measure relative level of brain function in deficient and reversed rats would be of interest, but was beyond the scope of our this project.

In conclusion, complete single or multiple nutrient deficiencies may be rare in the wild. However, the body has adaptive mechanisms to enable an organism to survive severe conditions for short periods until favorable conditions return. We surmise from our results that these changes may be adaptive in nature; providing mechanisms by which an organism can survive until favorable conditions return. While the physical health of the organism may deteriorate under these conditions, the organism is capable of surviving and as shown in the choline, folic acid, and vitamin B12 deficiency studies, can recover rapidly. However, the period of severe deficiency may have long-term health ramifications. This also has implications for the human population.

Whether self-initiated through poor dietary choices or environmental, nutrient deprivation may have short-term and long-term health ramifications as well. These could lead to states which might be cancer initiating such as those seen in the rat; but may also lead to states which might initiate other forms of degenerative disease. More studies should be done to make clearer links between disease initiation and particular nutrients. However, we feel that these results show clearly that maintaining adequate nutrient levels is a key to maintaining health.

\section{Acknowledgements}

The research was supported by the Center for the Improvement of Human Functioning International. Data analysis and completion of the manuscript were supported by Allan P. Markin.

\section{REFERENCES}

[1] C. Funk, “The Vitamins," Williams and Wilkins, Baltimore, 1922.

[2] V. Tanphaichitr , “Thiamin,” In: M. E. Shils, J. A. Olsen and M. Shike, Eds., Modern Nutrition in Health and Disease, Williams and Wilkins, Baltimore, 1999, pp. 381389.

[3] G. Rindi, “Thiamin,” In: E. E. Ziegler and L. J. Filer, Eds., Present Knowledge in Nutrition, ILSI Press, Washington DC, 1996, pp. 160-166.

[4] T. Brody, "Nutritional Biochemistry," 2nd Edition, Academic Press, San Diego, 1999.

[5] Micronutrient Information Center, Linus Pauling Institute at Oregon State University, 2012.

http://lpi.oregonstate.edu/infocenter/vitamins/

[6] D. Cervantes-Laurean, N. G. McElvaney and J. Moss, "Niacin,” In: M. Shils, J. A. Olson, M. Shike and A. C. Ross, Eds., Modern Nutrition in Health and Disease, Williams and Wilkins, Baltimore, 1999, pp. 401-411.

[7] P. C. Fry, H. M. Fox and H. G. Tao, "Metabolic Response to a Pantothenic Acid Deficient Diet in Humans,” Journal of Nutritional Science and Vitaminology, Vol. 22, No. 4, 1976, pp. 339-346. doi:10.3177/jnsv.22.339

[8] D. B. McCormick, "Vitamin B6," In: B. A. Bowman and R. M. Russell, Eds., Present Knowledge in Nutrition, Vol. 1, International Life Sciences Institute, Washington DC, 2006, pp. 269-277.

[9] J. E. Leklem, "Vitamin B6,” In: L. Machlin, Ed., Handbook of Vitamins, Marcel Decker Inc., New York, 1991, pp. 341-378.

[10] S. Dakshinamurti and K. Dakshinamurti, "Vitamin B6," In: J. Zempleni, R. B. Rucker, D. B. McCormick and J. W. Suttie, Eds., Handbook of Vitamins, CRC Press (Taylor \& Fracis Group), New York, 2007, pp. 315-359.

[11] J. E. Leklem, “Vitamin B6,” In: M. Shils, J. A. Olson, M. Shike and A. C. Ross, Eds., Modern Nutrition in Health and Disease, Williams \& Wilkins, Baltimore, 1999, pp. 413-422.

[12] A. D. Mackey, S. R. Davis and J. F. Gregory III, "Vitamin B6,” In: M. E. Shils, M. Shike, A.C. Ross, B. Caballero and R. J. Cousins, Eds., Modern Nutrition in Health and Disease, Williams and Wilkins, Philadelphia, 2006, pp. 452-461.

[13] N. J. Wald, M. R. Law, J. K. Morris and D. S. Wald, "Quantifying the Effect of Folic Acid," Lancet, Vol. 358, No. 9298, 2001, pp. 2069-2073. doi:10.1016/S0140-6736(01)07104-5

[14] L. B. Bailey and J. F. Gregory III, "Polymorphisms of Methylenetetrahydrofolate Reductase and Other Enzymes: Metabolic Significance, Risks and Impact on Folate Requirement,” Journal of Nutrition, Vol. 129. No. 5, 1999, pp. 919-922.

[15] H. McNulty, G. J. Cuskelly and M. Ward, "Response of Red Blood Cell Folate to Intervention: Implications for Folate Recommendations for the Prevention of Neural Tube Defects," American Journal of Clinical Nutrition, Vol. 7, No. 5, 2000, pp. 1308S-1311S. 
[16] S. W. Choi and J. B. Mason, "Folate and Carcinogenesis: An Integrated Scheme,” Journal of Nutrition, Vol. 130. No. 2, 2000, pp. 129-132.

[17] B. Shane, “Folic Acid, Vitamin B12, and Vitamin B6,” In: M. Stipanuk, Ed., Biochemical and Physiological Aspects of Human Nutrition, W.B. Saunders Co., Philadelphia, 2000, pp. 483-518.

[18] Food and Nutrition Board, Institute of Medicine, "Vitamin B12. Dietary Reference Intakes: Thiamin, Riboflavin, Niacin, Vitamin B6, Vitamin B12, Pantothenic Acid, Biotin, and Choline," National Academy Press, Washington DC, 1998, pp. 306-356.

[19] V. Herbert, “Vitamin B12,” In: E. E. Ziegler and L. J. Filer, Eds., Present Knowledge in Nutrition, 7th Edition, ILSI Press, Washington DC, 1996, pp. 191-205.

[20] P. G. Reeves, F. H. Nielsen and G. C. Fahey Jr., “AIN-93 Purified Diets for Laboratory Rodents," Journal of Nutrition, Vol. 123, No. 11, 1993, pp. 1939-1951.

[21] A. Odynets, "Beddings of Laboratory Animals: Criteria of Biological Evaluation,” Lab. Zyhvotnye, Vol. 1, No. 3, 1991, pp. 70-76.

[22] A. J. Clifford, D. S. Wilson and N. D. Bills, "Repletion of Folate-Depleted Rats with an Amino Acid-Based Diet Supplemented with Folic Acid,” Journal of Nutrition, Vol. 119, No. 12, 1989, pp. 1956-1961.

[23] D. Nakae , H. Yoshiji, Y. Mizumoto , K. Horiguchi , K. Shiraiwa , K. Tamura, A. Denda and Y. Konishi, "High Incidence of Hepatocellular Carcinoma Induced by a Choline Deficient L-Amino Acid Defined Diet in Rats,” Cancer Research, Vol. 52, No. 18, 1992, pp. 5042-5045.

[24] D. Nakae, H. Yoshiji, H. Maruyama, T. Kinugasa, A. Denda and Y. Konishi, "Production of Both 8-Hydroxydeoxyguanosine in Liver DNA and y-Glutamyltransferase-posistive Hepatocellular Lesions in Rats Given a Choline-deficient, L-Amino Acid-defined
Diet,” Journal of Cancer Research, Vol. 81, 1990, pp. 1081-1084.

[25] N. Sawada, L. Poirier, S. Moran, Y. H. Xu and H. C. Pitot, "The Effect of Choline and Methionine Deficiencies on the Number and Volume Percentage of Altered Hepatic Foci in the Presence or Absence of Diethylnitrosamine Initiation in Rat Liver," Carcinogenesis (Lond.), Vol. 11, No. 2, 1990, pp. 273-281. doi:10.1093/carcin/11.2.273

[26] J. C. Gonder and K. Laber, “A Renewed Look at Laboratory Rodent Housing and Management," Institute of Laboratory Animal Resources Journal, Vol. 48, No. 1, 2007, pp. 29-36.

[27] R. H. Weichbrod, C. F. Cisar, J. G. Miller, R. C. Simmonds, A. P. Alvares and T. H. Ueng, "Effects of Cage Beddings on Microsomal Oxidative Enzymes in Rat Liver,” Laboratory Animal Science, Vol. 38, No. 3, 1988, pp. 296-298.

[28] M. Maeno, Y. Morimoto, T. Hayakawa, Y. Suzuki and H. Tsuge, "Feeding Experiments of Pyridoxine Derivatives as Vitamin B6," International Journal for Vitamin and Nutrition Research, 1997, Vol. 67, No. 6, pp. 444-449.

[29] K. Dakshinamurti, S. K. Sarma and D. Bonke, "Influence of B Vitamins on Binding Properties of Serotonin Receptors in the CNS of Rats," Klinische Wochenschrift, Vol. 68, No. 2, 1990, pp. 142-145. doi:10.1007/BF01646863

[30] B. C. Richardson, "Methylation in the Regulation of Cell Function: Autoimmunity, Aging, and Cancer,” Journal of Nutrition, Vol. 132, No. 8, 2002, pp. 2401S-2405S.

[31] J. F. Costello and C. Plass, "Methylation Matters,” Journal of Medical Genetics, Vol. 38, No. 5, 2001, pp. 285303. doi:10.1136/jmg.38.5.285

[32] C. Amoreira, W. Hindermann and C. Grunau, "An Improved Version of the DNA Methylation Database (MethDB)," Nucleic Acids Research, Vol. 31, No. 1, 2003, pp. 75-77. doi:10.1093/nar/gkg093 